\title{
A search for water maser emission from post-AGB stars
}

\author{
J. M. Chapman ${ }^{1}$, R. M. Deacon ${ }^{2}$, A. J. Green ${ }^{2}$, and M. Cohen ${ }^{3}$ \\ ${ }^{1}$ Australia Telescope National Facility, PO Box 76, Epping, NSW 1710, Australia \\ ${ }^{2}$ School of Physics A29, University of Sydney, NSW 2006, Australia \\ ${ }^{3}$ Radio Astronomy Laboratory, University of California, Berkeley, CA 94720, USA
}

\begin{abstract}
We have used the Tidbinbilla 70-m antenna to search for $22 \mathrm{GHz} \mathrm{H}_{2} \mathrm{O}$ maser emission from a sample of 85 evolved stars. 21 detections were made. Of these 15 were from massive AGB stars. High-velocity $\mathrm{H}_{2} \mathrm{O}$ maser emission was detected from five sources, of which four are post-AGB stars. Three of the high-velocity sources, b292 (IRAS 18043-2116), d46 (IRAS 154455449), and d62 (IRAS 15544-5332) were new discoveries. d46 is also a source of non-thermal radio continuum emission. The high-velocity $\mathrm{H}_{2} \mathrm{O}$ maser emission and the radio continuum from post-AGB stars are probably associated with shocks that form from wind-wind interactions.
\end{abstract}

Keywords. masers, stars: AGB and post-AGB, evolution, mass-loss, radio continuum

\section{Introduction}

Post-asymptotic giant branch (post-AGB) stars are in a short-lived evolutionary stage between the AGB and planetary nebulae. During this stage the regular long-period pulsations of AGB stars cease and the stars change from losing mass in slow dense winds, with wind expansion velocies of typically $5-15 \mathrm{~km} \mathrm{~s}^{-1}$, to losing mass in hot low-density winds with wind velocities of several hundreds kilometres per second.

Masers provide an excellent means of studying mass-loss from AGB and post-AGB stars. $\mathrm{OH}$ maser emission at $1612 \mathrm{MHz}$ has been detected from the outer circumstellar envelopes of more than 1500 AGB stars. In most cases the $\mathrm{OH} 1612 \mathrm{MHz}$ spectra are double-peaked, consistent with spherical, or near-spherical outflows. In contrast, planetary nebulae often show complex and diverse morphologies with elliptical, bipolar and filamentary structures observed. It is likely that changes in the envelope morphologies begin during the early post-AGB stage.

We are studying the maser properties of a well-defined sample of 85 evolved stars selected from the $\mathrm{OH} 1612 \mathrm{MHz}$ Galactic Plane survey of Sevenster et al. (2001 and references therein). The sources were selected to be post-AGB candidate stars on the basis of their IRAS and/or MSX far-infrared colours. The $\mathrm{OH} 1612,1665$ and $1667 \mathrm{MHz}$ properties of the sources, and the full selection criteria are discussed by Deacon et al. (2004). Here we discuss the results of a search for $\mathrm{H}_{2} \mathrm{O}$ maser emission.

Figure 1 shows the MSX colour-colour plot for the sources in our sample with good quality MSX fluxes. Following Sevenster (2002a,b) the plot is divided into four quadrants. Post-AGB stars are located in quadrants I (late-type or older sources) and IV (early-type or younger sources). The sources seen in quadrant III are known as 'LI' stars and have an excess of emission at $60 \mu \mathrm{m}$ emission compared to AGB stars with little or no $\mathrm{OH}$ $1612 \mathrm{MHz}$ maser emission. On the standard IRAS [12-25], [25-60] colour-colour plot, they are seen to the left of the well-known evolutionary track for AGB stars (van der Veen \& Habing 1988). Our sample included 30 LI sources and 55 sources with the MSX colours of post-AGB stars. 


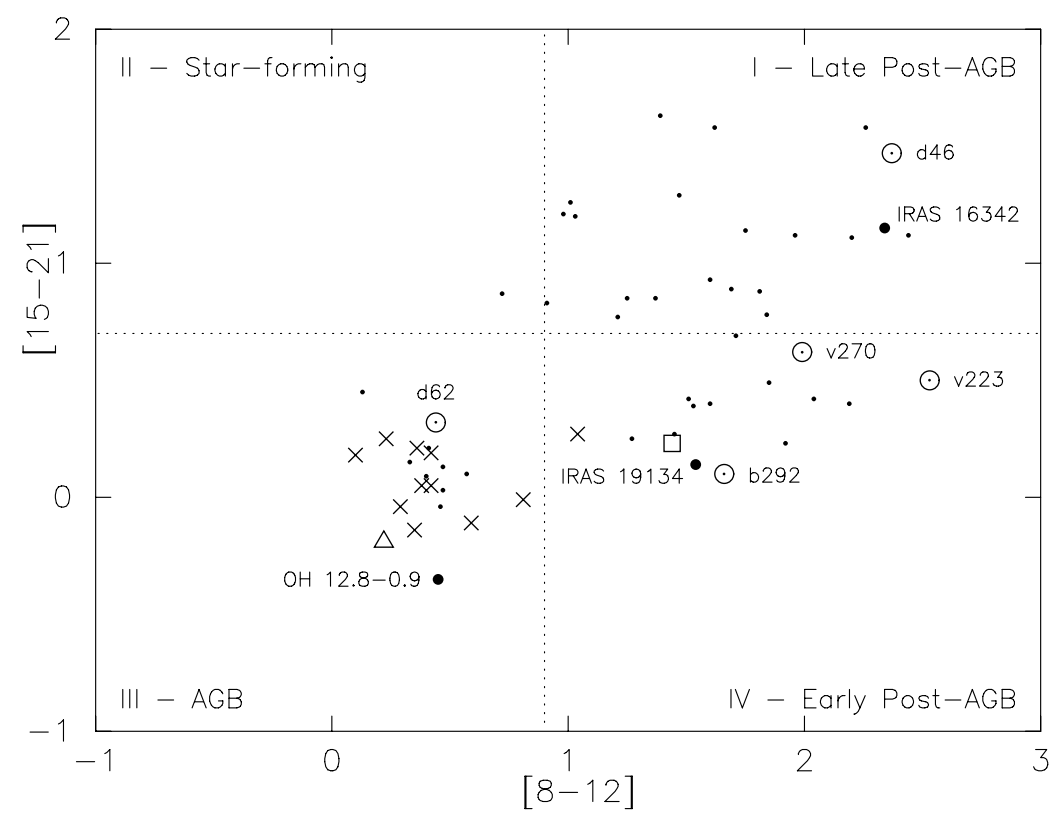

Figure 1. MSX colour-colour plot for sources in the sample. Sources with no detected $\mathrm{H}_{2} \mathrm{O}$ emission are shown as small dots. Sources with regular $\mathrm{H}_{2} \mathrm{O}$ maser profiles are shown as crosses. The dotted circles indicate five high-velocity $\mathrm{H}_{2} \mathrm{O}$ maser sources detected with the Tidbinbilla antenna. Three high-velocity sources with previously published detections (IRAS 19134+2131, IRAS 16342-3814 and $\mathrm{OH} \mathrm{12.8-0.9)} \mathrm{are} \mathrm{shown} \mathrm{as} \mathrm{large} \mathrm{dots} \mathrm{(Deacon} \mathrm{et} \mathrm{al.} \mathrm{2007).} \mathrm{All} \mathrm{the}$ high-velocity $\mathrm{H}_{2} \mathrm{O}$ maser sources are labelled.

Observations at a rest frequency of $22.235 \mathrm{GHz}$ were taken between 2003 May and 2004 April with the Tidbinbilla 70-m DSS 43 antenna. Each of the 85 sources was observed for a total on-source integration time of 7 minutes, using a bandpass of $16 \mathrm{MHz}$ and 8193 channels, corresponding to a total velocity coverage of $\sim 200 \mathrm{~km} \mathrm{~s}^{-1}$. The spectra were Hanning smoothed to give a velocity resolution of $0.18 \mathrm{~km} \mathrm{~s}^{-1}$. The antenna had a pointing accuracy of about 7 arcseconds. The one-sigma noise levels in the spectra were between 40 and 120 mJy. Repeated observations were taken in a few cases to confirm weak detections.

\section{The LI sources}

We detected $\mathrm{H}_{2} \mathrm{O}$ maser emission from 21 of the 85 sources in the sample. Of these, 15 detections were from the LI sources. In 14/15 cases, the $\mathrm{H}_{2} \mathrm{O}$ maser emission was detected at velocities that were equal to, or within, the $\mathrm{OH} 1612 \mathrm{MHz}$ velocity ranges. A typical example is shown in Figure 2. Such double-peaked $\mathrm{H}_{2} \mathrm{O}$ profiles are characteristic of AGB stars with high mass-loss rates where the $\mathrm{H}_{2} \mathrm{O}$ molecules are located closer to the central stars than the $\mathrm{OH}$ molecules. The $\mathrm{OH} 1612 \mathrm{MHz}$ spectra of the LI sources are almost invariably doubled-peaked indicating spherical or near-spherical circumstellar envelopes. For one LI source in the present sample, d62 (IRAS 15544-5332), the $22 \mathrm{GHz}$ maser spectrum showed a single narrow peak located outside of the $\mathrm{OH}$ velocity range, indicating a possible high-velocity outflow.

The evolutionary status of LI stars as AGB or post-AGB stars has been uncertain. From their average Galactic latitude of 0.46 degrees, Sevenster $(2002 \mathrm{a}, \mathrm{b})$ determined that they are massive stars with an average mass of about $4 \mathrm{M}_{\odot}$. From their Galactic 

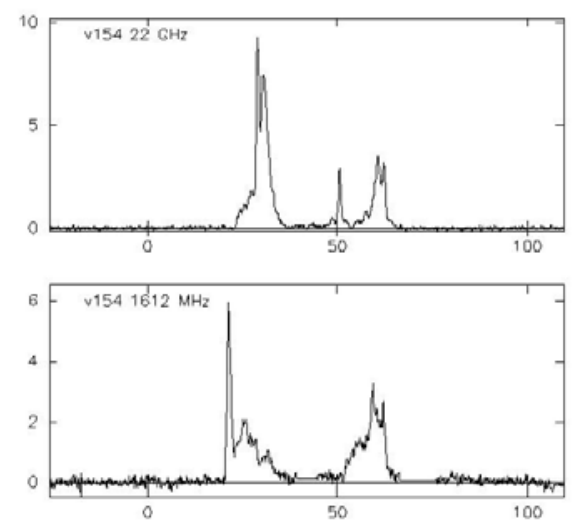

Figure 2. $22 \mathrm{GHz} \mathrm{H}_{2} \mathrm{O}$ (top) and $\mathrm{OH} 1612 \mathrm{MHz}$ (bottom) maser spectra for the LI source, v154 (IRAS 18327-0715). The stellar velocity, determined from the $\mathrm{OH} 1612 \mathrm{MHz}$ profile is $40.5 \mathrm{~km} \mathrm{~s}^{-1}$.

distribution and their $\mathrm{OH}$ and $\mathrm{H}_{2} \mathrm{O}$ properties we infer they are massive stars with high mass-loss rates that are still AGB stars. As such they are the precursors to the most massive post-AGB stars and planetary nebulae.

Cohen et al. (2006) have discussed the radio properties of a remarkable source, V1018 Sco, where a faint ionised nebula is seen centred on an AGB star with a pulsation period of $\sim 1460$ days. This source has MSX colours similar to the LI sources in our sample. Radio continuum emission has been detected from two locations from within the nebula. The stronger continuum feature (their feature A) has a spectral index of -0.8 between 3 and $13 \mathrm{~cm}$, consistent with non-thermal synchroton radio emission.

\section{High-velocity water sources}

We detected $\mathrm{H}_{2} \mathrm{O}$ maser emission at velocities outside of the $\mathrm{OH}$ velocity range from five sources. One source, d62, is an LI star while the other four are post-AGB stars (Figure 1).

Two sources, v223 (W43A) and v270 (IRAS 18596+0315) were previously known. W43A belongs to a rare group of sources known as 'water fountains'. In these sources $\mathrm{H}_{2} \mathrm{O}$ maser emission is detected over a large velocity range from collimated bipolar jets. The jets have very short dynamical ages of typically 100 years. From polarisation observations of the $\mathrm{H}_{2} \mathrm{O}$ maser emission, Vlemmings, Diamond \& Imai (2006) have shown that the jets in W43A are likely to be magnetically collimated. Our $22 \mathrm{GHz}$ spectrum of v270 (not shown) shows a symmetric $\mathrm{H}_{2} \mathrm{O}$ maser profile with emission over two fairly narrow velocity ranges, each approximately $30 \mathrm{~km} \mathrm{~s}^{-1}$ from the stellar velocity, at twice the expansion velocity of the $\mathrm{OH} 1612 \mathrm{MHz}$ maser emission. We consider that v270 is a likely water fountain source, although high angular resolution images are needed to confirm this.

\section{b292, a new water fountain source}

We discovered extremely broad $\mathrm{H}_{2} \mathrm{O}$ maser emission from the source b292 (IRAS 180432116). As shown in Figure 3, numerous emission features were detected over velocities between -20 and $185 \mathrm{~km} \mathrm{~s}^{-1}$. It is possible that other features are present beyond the range of observed velocities. The stellar velocity is known from the $\mathrm{OH} 1612 \mathrm{MHz}$ spectrum to be $\sim 83 \mathrm{~km} \mathrm{~s}^{-1}$. The strongest $\mathrm{H}_{2} \mathrm{O}$ emission was detected at velocities between $\sim 95$ and $120 \mathrm{~km} \mathrm{~s}^{-1}$, corresponding to the far side of the star. b292 is the 


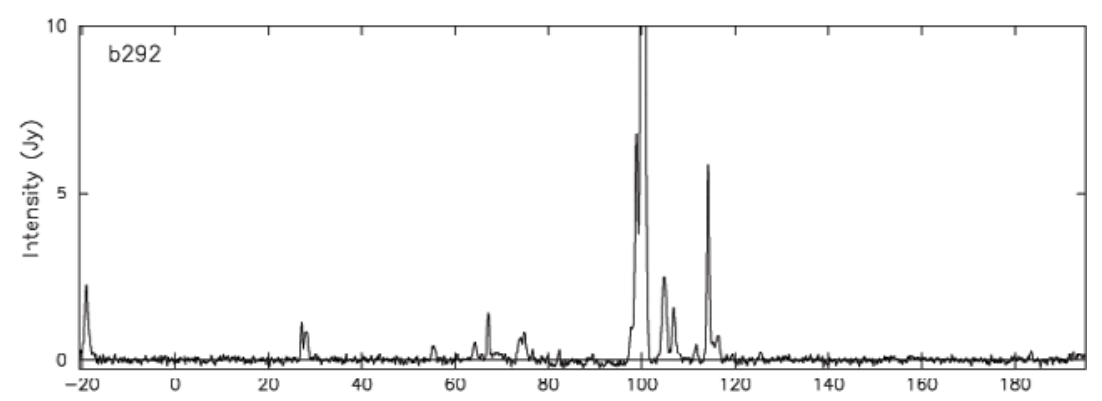

Figure 3. $\mathrm{H}_{2} \mathrm{O}$ maser spectrum of b292 (IRAS 18093-2116) obtained with the Tidbinbilla 70-m antenna in 2004 March. The spectrum is truncated in intensity to show the weak features.

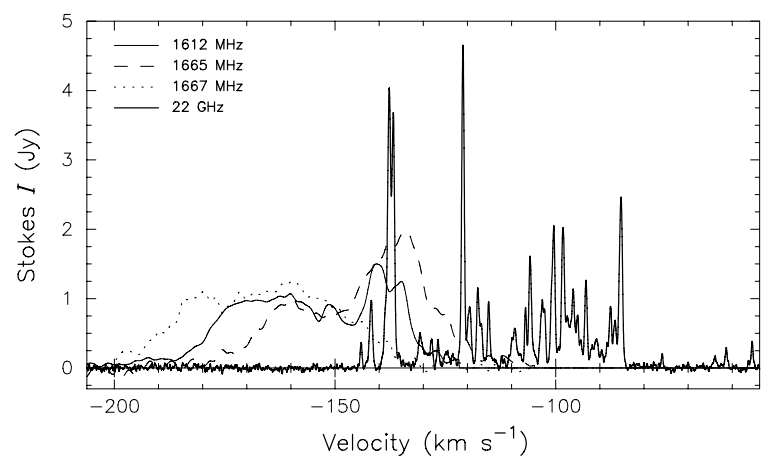

Figure 4. OH 1612, 16655, and $1667 \mathrm{MHz}$ maser spectra of the post-AGB star d46 (IRAS 15445-5449), are shown together with the Tidbinbilla $22 \mathrm{GHz} \mathrm{H}_{2} \mathrm{O}$ maser spectrum (from Deacon et al. 2007).

only post-AGB star to have been detected in the $\mathrm{OH} 1720 \mathrm{MHz}$ transition (Sevenster \& Chapman 2001) and this emission is thought to arise behind a shock front associated with a slow-wind - fast-wind collision. From the $\mathrm{H}_{2} \mathrm{O}$ maser spectrum, the $\mathrm{OH} 1720$ $\mathrm{MHz}$ detection and the stellar classification as a young post-AGB star (Figure 1) we classify this as a water fountain source.

\section{The peculiar source $d_{4} 6$}

Figure 4 shows the Tidbinbilla $\mathrm{H}_{2} \mathrm{O}$ maser spectrum for $\mathrm{d} 46$ together with $\mathrm{OH}$ spectra from Deacon et al. (2004). This source has unusual maser profiles with emission detected over a total velocity range of approximately $150 \mathrm{~km} \mathrm{~s}^{-1}$. The $\mathrm{OH}$ profiles at 1612,1665 and $1667 \mathrm{MHz}$ are all broad and similar in shape. However, the $\mathrm{OH} 1665 \mathrm{MHz}$ emission is red-shifted relative to the $\mathrm{OH} 1612 \mathrm{MHz}$ emission while the $1667 \mathrm{MHz}$ emission is blueshifted. The stellar velocity is unknown but the $\mathrm{OH}$ spectra suggest a systemic velocity near $-150 \mathrm{~km} \mathrm{~s}^{-1}$. The $22 \mathrm{GHz} \mathrm{H}_{2} \mathrm{O}$ spectrum is red-shifted by about $50 \mathrm{~km} \mathrm{~s}^{-1}$ relative to the $\mathrm{OH}$ maser emission with numerous narrow features detected at velocities between -150 and $-50 \mathrm{~km} \mathrm{~s}^{-1}$. As with b292, other features outside of the observed velocity range could be present. The MSX colours indicate that $\mathrm{d} 46$ is a more highly evolved post-AGB star than b292 (Figure 1) while the complex and asymmetric maser profiles indicate a highly disturbed outflow.

Figure 5 shows a radio continuum spectrum of d46 from observations at 3, 6 and 13 $\mathrm{cm}$ taken with the Australia Telescope Compact Array in 1998. The radio continuum spectrum of the LI star V1018 Sco at wavelengths between 3 and $36 \mathrm{~cm}$ is also shown (Feature A, see Cohen et al. 2006). For both sources the radio continuum spectrum has a 


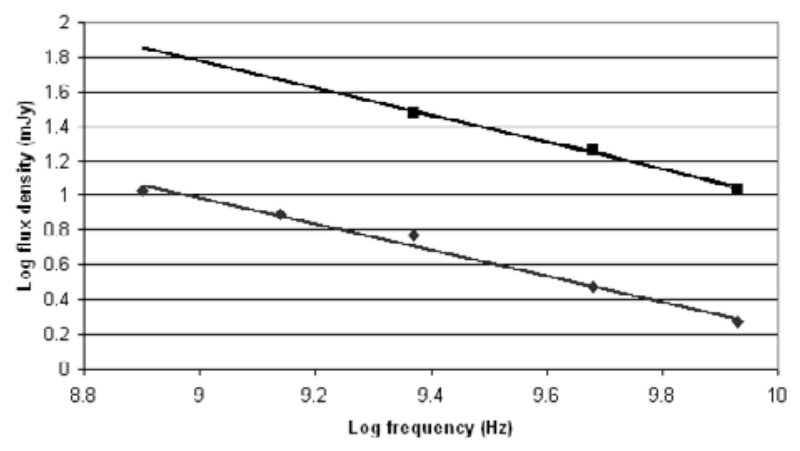

- V1018 Sco: A - —-D46: 1998 — Linear (D46: 1998) —-Linear (V1018 Sc0: A)

Figure 5. Radio continuum spectra for d46 (top) and the LI source V1018 Sco (bottom). In both cases a linear fit to the data gives a spectral index of -0.8 (adapted from Cohen et al. 2006).

spectral index of $\alpha=-0.8$ (defined as $S_{\nu} \propto \nu^{\alpha}$ ), consistent with synchroton radio emission. We interpret this as evidence for shocks due to a wind-wind interaction between the hot wind that turns on as the star leaves the AGB, and the AGB circumstellar envelope.

\section{Discussion and conclusions}

From this survey of 85 sources, we detected two different types of $\mathrm{H}_{2} \mathrm{O}$ maser emission. AGB-type $\mathrm{H}_{2} \mathrm{O}$ maser profiles were detected from $50 \%$ of the 30 LI sources in our sample. This is consistent with an identification of these objects as massive AGB stars. We also detected five sources with high-velocity $\mathrm{H}_{2} \mathrm{O}$ emission. Four of these were post-AGB stars. We did not detect any 'regular' $\mathrm{H}_{2} \mathrm{O}$ maser profiles from sources with very red IRAS or MSX colours. We surmise that the $\mathrm{H}_{2} \mathrm{O}$ maser emission that is seen during the AGB evolutionary stage, disappears as a star evolves away from the AGB. However, a new source of $\mathrm{H}_{2} \mathrm{O}$ maser emission, associated with high-velocity jets, may appear. The detection of high-velocity $\mathrm{H}_{2} \mathrm{O}$ emission in two LI sources (d62 and OH12.8-0.9, see Figure 1) suggests that for some massive stars the jet outflows may start before the star has left the AGB.

\section{References}

Cohen, M., Chapman, J. M., Deacon, R. M., Sault, R. J., Parker, Q. A., \& Green, A. J. 2006, MNRAS 369, 189

Deacon, R. M., Chapman, J. M. \& Green, A. J. 2004, ApJS 155, 595

Deacon, R. M., Chapman, J. M., Green, A. J., \& Sevenster, M. N. 2007, ApJ 658, 1096

Sevenster, M. N., van Langevelde, H. J., Moody, R. A., Chapman, J. M., Habing, H. J., \& Killeen, N. E. B. 2001, $A \mathscr{E} A, 366,481$

Sevenster, M. N. \& Chapman, J. M. 2001, ApJ, 546, L119

Sevenster, M. N., 2002a, AJ, 123, 2772

Sevenster, M. N., 2002b, AJ, 123, 2788

van der Veen, W. E. C. J. \& Habing, H. J. 1988, A\& A, 194, 125

Vlemmings, W. H. T., Diamond, P. J. \& Imai, H. 2006, Nature, 400, 58 